\title{
An Algorithm of Form Deviation Calculation in Coordinate Measurements of Free-Form Surfaces of Products
}

\author{
Marek Magdziak* \\ Rzeszow University of Technology, Faculty of Mechanical Engineering and Aeronautics, Poland
}

The article presents the new method of form deviation calculation in the case of coordinate measurements of free-form surfaces of workpieces. The proposed algorithm of form deviation evaluation is based on the interpolation process of corrected measured points. Corrected measured points are the results of probe radius correction process applied in the coordinate measuring technique. The interpolation is conducted by using of the Lagrange and Chebyshev methods. The developed algorithm of deviation calculation can be implemented in most commercial software cooperating with a coordinate measuring machine. In the article the results of numerical and experimental investigations connected with the proposed algorithm are presented. The numerical investigations concern the simulation of contact coordinate measurements of products, which can be conducted on a coordinate measuring machine. The measured products are composed of free-form surfaces characterized by various curvature and consequently various degree of geometric complexity. The experimental investigations were conducted in order to verify the results of simulation studies. The presented method improves the accuracy of measurements of free-form surfaces of products.

Keywords: coordinate measuring technique, free-form surface, interpolation, form deviation

Highlights

- The new method of form deviation calculation is presented.

- The algorithm is based on the interpolation of points by using of the Lagrange and Chebyshev methods.

- The shown algorithm improves the accuracy of measurements of free-form surfaces.

- The new method is compared to existing algorithms of form deviation assessment.

- $\quad$ The developed algorithm can be implemented in measurement software.

\section{INTRODUCTION}

The coordinate measuring technique plays very important role in modern manufacturing processes of products. That technique can be used for example in reverse engineering processes, where the coordinate measuring technique can be used in order to digitize a product. The digitalization is performed at the beginning of a reverse engineering process of an object. Workpieces measured using the coordinate measuring technique can be produced by means of machining or rapid prototyping techniques. Measured products can be characterized by regular geometric shapes or can be composed of free-form surfaces [1] to [3]. Measurements of free-form surfaces can be divided into data acquisition and data processing [4]. The article concerns issues connected with the second stage of measurements - data analyzing.

Free-form surfaces are used in automotive and aerospace industries [5]. The examples of products with free-form surfaces, which can be measured using the coordinate measuring technique, are car body parts, turbine blades of aircraft engines and optic elements used in imaging systems [5] and [6]. The paper [5] contains the classification of free-form shape applications. The results of measurements of a machined free-form mould insert of an optical lens are presented in the paper [4]. The measurements were conducted by using of the intrinsic feature-based pattern analysis method dedicated to measuring ultraprecision free-form surfaces. Savio et al. [7] presented the results of measurements of a turbine blade. The aim of investigations was to establish the traceability of free-form measurements on coordinate measuring machines. The analyzed turbine blade was measured by using of different parameters associated with, for example, scanning speed and points density.

There can be distinguished a number of coordinate measuring gages, which can be used during coordinate measurements of curvilinear surfaces of most measured products. The examples of such measuring instruments are [5] and [8]:

- contact coordinate measuring machines (CMMs),

- redundant coordinate measuring gages (coordinate measuring arms, laser trackers),

- non-contact coordinate measuring machines (CMMs equipped with non-contact probing systems, measuring machines based on the photogrammetry, the laser triangulation or the computed tomography).

For measuring free-form optics very accurate coordinate measuring systems should be used. In the 
case of contact measurements CMMs with a resolution in nanometer range should be applied. Moreover, optical objects composed of free-form surfaces can be measured by using of probing systems mounted in ultra-precision manufacturing machines [6].

The accuracy of measurements with the use of above mentioned coordinate measuring gages can be influenced by the following factors:

- geometrical deviations of coordinate measuring instruments,

- deviations of measured products,

- environmental conditions in quality control departments,

- the inaccuracy of a probing system,

- applied measurement strategies,

- available algorithms included in measurement software cooperating with e.g. CMMs.

That factors are the sources of coordinate measurement uncertainty. Some of the mentioned factors are included in the standard [9], which concerns the method of determining the uncertainty of a coordinate measurement with the use of calibrated workpieces or measurement standards. The article contains an analysis of some issues connected with a measurement strategy and the inaccuracy of algorithms which are the part of commercial software of coordinate measuring gages.

Measurement software of modern coordinate measuring instruments may include, for example, various methods of probe radius correction process. That algorithms are intended to the calculation of coordinates of corrected measured points on the basis of coordinates of indicated measured points, which represent the center of a stylus tip of a contacting probing system. The corrected measured points should represent, as much as it is possible, the real shape of measured objects. The process of probe radius correction is the basis for contact coordinate measurements with the use of coordinate measuring machines or coordinate measuring arms. The real shape of a measured object has significant impact on the accuracy of coordinate measurements of free-form surfaces of workpieces. The wrong direction along which the probe radius correction process is conducted leads to the calculation of wrong coordinates of corrected measured points and consequently wrong measurement data [5]. There can be distinguished a large number of probe radius correction methods.

Liang and Lin [10] proposed the method for probe radius correction of a measuring probe stylus tip in the case of measuring curvilinear surfaces of products. That method consists in determining the set of triangles defined by indicated measured points localized on adjacent measurement paths. The unit normal vector which is being sought for a given point is computed in result of vector multiplication of vectors representing two sides of related triangle.

The authors of the paper [11] presented a method for determining the direction of probe radius correction based on measurements of two groups of points, each of them containing two points located at the other sides of a given indicated measured point at which a correction vector is being sought. Each group of points represents an individual, different direction of a measured surface and defines some individual vector. The direction for probe radius correction is computed using vector multiplication of already generated vectors determined from the information about the above mentioned two groups of points.

The references [12] and [13] present a method for computing a corrected measured point in the case of measurements of curves with a scanning measuring probe. The related algorithm uses subsequent locations of a measuring probe stylus tip working in a scanning mode. Based on those subsequent locations there is created an approximate outline of a measured profile by joining arcs being parts of a measuring stylus tip in its successive locations. The approximated corrected measured point, associated with an indicated measured point in a given location and setting of a measuring probe, is computed in the middle of an arc being a segment of the approximated outline of a measured profile.

The mentioned methods of a probe radius correction process are focused on identifying corrected measured points and do not take into account the algorithms of calculation of form deviations of measured workpieces, which can be composed of free-form surfaces. In the article the new method of assessing the accuracy of curvilinear surfaces of workpieces is presented. The proposed method may supplement developed methods of computing of corrected measured points.

In the next parts of the article selected existing methods of assessing form deviations, which are available in the chosen commercial software of a $\mathrm{CMM}$ and the results of performed investigations are presented. The simulation investigations concern the comparison of selected algorithms of product accuracy verification. The proposed method was compared to the existing methods of calculation of form deviations, which are available in the selected commercial software cooperating with a coordinate measuring machine. The simulation investigations were conducted with the use of arbitrarily selected workpieces containing free-form surfaces. On the 
basis of gained results of performed research the statistical analysis was done.

The experimental investigations were conducted in order to verify the gained results of numerical research. They were performed by using of a selected coordinate measuring machine and different measurement parameters. The last parts of the paper contain the discussion and the conclusions regarding the results of conducted investigations and the possibility of using the proposed method of form deviation calculation in the coordinate measuring technique.

\section{THE EXISTING METHODS OF ASSESING FORM DEVIATIONS}

There is a large number of measurement software cooperating with coordinate measuring gages. In the second section of the article the existing methods of assessing form deviations are described in the case of the widely used Calypso measurement software, which is produced by the Carl Zeiss company.

The user of the considered measurement software is able to compute the values of a form deviation of free-form surfaces, which are the part of a measured object, by using of the following methods of form deviation calculation [14]:

- a deviation in a nominal vector direction a local deviation is measured at each nominal point in the direction of a normal vector up to the intersection with an actual curve, which represents corrected measured points (the method denoted as the first method in the next parts of the article);

- an actual-nominal deviation - a local deviation is measured as the distance between the point located on an actual curve and a nominal curve (the method denoted as the second method).

The described methods of form deviation calculation were used during the investigations. Based on the recommendations of the producer the user of the measurement software should use in many measuring applications in the coordinate measuring technique mainly the first method of form deviation calculation. However, in that method, when measured free-form surfaces have the shape significantly different from the nominal one, the results of calculation of a form deviation substantially depend on the number of measured points [14].

In the case of the first method the point lying on a measured curve, which represents the real form of a measured product, is not the same point whose coordinates are registered by using of a coordinate measuring machine. The number of measured points in the first method of form deviation calculation is different than the number of points measured by using of a CMM. Similarly in the case of form deviation calculation based on measured points in the second method the number of nominal points does not correspond to the number of points, which is used in the case of the first method of form deviation calculation (Fig. 1). The information about the coordinates of both nominal and measured points is available in the $2 \mathrm{D}$ curve and $3 \mathrm{D}$ curve measurement elements. When increasing the number of measured points the accuracy of calculation of form deviations based on nominal points in the first method is getting higher. Unfortunately, the drawback of using this measurement software is that the user has no information about the type of a free-form curve which approximates measured points.

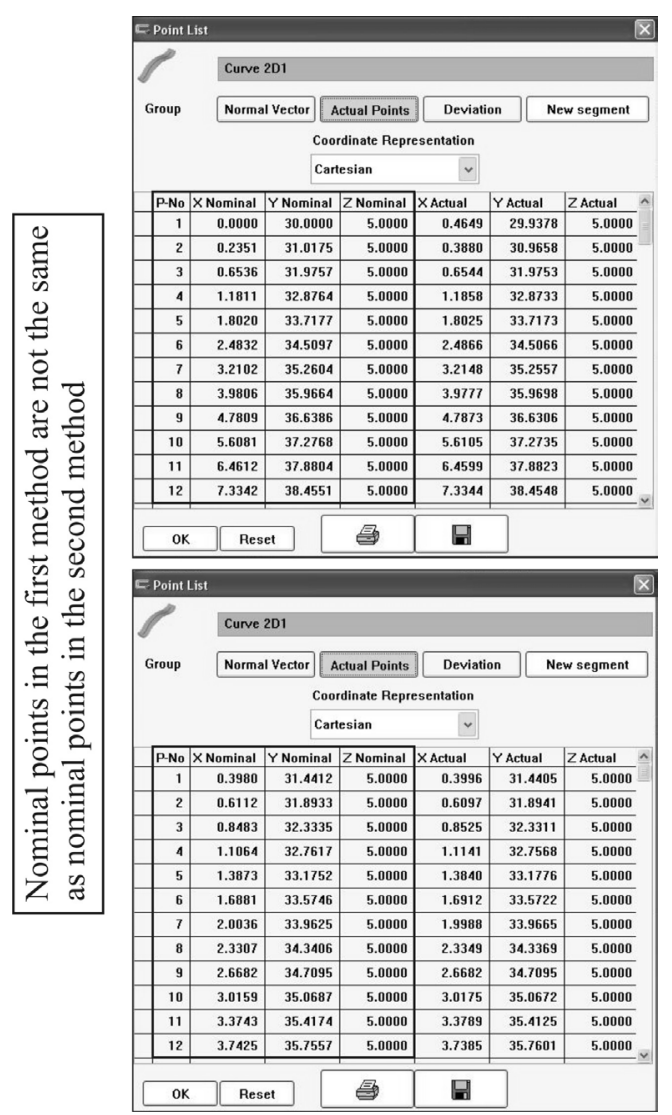

Fig. 1. The coordinates of nominal points calculated by using of the first and the second methods

\section{THE NEW METHOD OF FORM DEVIATION CALCULATION}

The new method of form deviation calculation of freeform surfaces of products is based on the evaluation of a local deviation, similar to the first method, 
at nominal points of a measured workpiece. The application of the proposed method is presented on the basis of the Calypso measurement software. The considered method requires the information included in the 2D curve and 3D curve measurement elements, which are available in that software. The required information is exported from the above mentioned elements during the calculation of a form deviation using the first and the second methods. However, the new method can be implemented not only in the Calypso software. The data necessary for the proposed algorithm can be obtained from most measurement software.

The usage of the type of a measurement element depends most of all on the shape of a measured object. The 2D curve and 3D curve measurement elements have different normal vectors perpendicular to a surface of a measured product at its nominal points. When using the 2D curve measurement element normal vectors lie in a single plane. Therefore, that element does not take into account the curvature of a measured surface around measured free-form curves. The 3D curve element includes the information about the shape of a measured curvilinear surface near the section plane used to create the considered measurement element. Consequently, the 3D curve measurement element has the possibility of using normal vectors not lying in a single plane. Moreover, the $3 \mathrm{D}$ curve element allows the use of a nonuniform distribution of nominal points along measured freeform profiles based on their curvature.

In the new method of form deviation calculation the information about the coordinates of nominal points is exported from the above mentioned measurement elements when using the first commercial method of deviation estimation. The coordinates of corrected measured points are imported to the proposed method of form deviation calculation when using the second method. The proposed method includes all measured points obtained by using of a coordinate measuring machine.

The new method of form deviation calculation enables the interpolation of corrected measured points by using of the Lagrange and Chebyshev methods. That method divides points measured by a coordinate measuring machine into groups composed of five measured points. Those groups are interpolated using two mentioned interpolation methods. Therefore, there are constructed the groups of polynomial curves of a fourth degree, which represent the real shape of a measured workpiece in its individual crosssections. In the next stage of the proposed method, after calculating interpolation curves, the deviations between nominal points and intersection points are calculated. The intersection points are created using interpolation curves and lines perpendicular to the measured profile of a workpiece at its nominal points. Therefore, the proposed method of form deviation estimation uses the information, apart from the coordinates of nominal and measured points, about normal vectors to free-form curves at nominal points. This information is taken from the first method of form deviation calculation. The user of the proposed method has the possibility of choosing the interpolation method of corrected measured points of free-form surfaces of products.

In the case of five interpolated measured points the interpolation function $F(x)$ and the base polynomials $T_{0}(x)-T_{4}(x)$ are written, using the Chebyshev interpolation method, with the following equations:

$$
\begin{aligned}
F(x)= & a_{0} T_{0}(x)+a_{1} T_{1}(x)+a_{2} T_{2}(x)+ \\
& +a_{3} T_{3}(x)+a_{4} T_{4}(x), \\
& \left\{\begin{array}{l}
T_{0}(x)=1 \\
T_{1}(x)=x \\
T_{2}(x)=2 x^{2}-1 \\
T_{3}(x)=4 x^{3}-3 x \\
T_{4}(x)=8 x^{4}-8 x^{2}+1
\end{array}\right.
\end{aligned}
$$

The interpolation function is defined in the interval $\langle a, b\rangle$. Therefore, the interpolation polynomial should be scaled using the following equation:

$$
x=\frac{2}{b-a} t-\frac{a+b}{b-a} .
$$

In the case of the Lagrange interpolation method and five interpolated corrected measured points with coordinates $\left(x_{i}, y_{i}\right)$ the interpolation function $L(x)$ is written with Eqs. (4) and (5).

$$
\begin{aligned}
L(x)= & y_{0} L_{0}(x)+y_{1} L_{1}(x)+y_{2} L_{2}(x)+ \\
& +y_{3} L_{3}(x)+y_{4} L_{4}(x) .
\end{aligned}
$$

The created method of form deviation calculation can be implemented as the new one in commercial software of a coordinate measuring machine. For example, the implementation can be done using the parameter coded measurements (PCM) module. That module has the possibility of using external applications, which take into account the proposed method. 


$$
\left\{\begin{array}{l}
L_{0}(x)=\frac{\left(x-x_{1}\right)\left(x-x_{2}\right)\left(x-x_{3}\right)\left(x-x_{4}\right)}{\left(x_{0}-x_{1}\right)\left(x_{0}-x_{2}\right)\left(x_{0}-x_{3}\right)\left(x_{0}-x_{4}\right)} \\
L_{1}(x)=\frac{\left(x-x_{0}\right)\left(x-x_{2}\right)\left(x-x_{3}\right)\left(x-x_{4}\right)}{\left(x_{1}-x_{0}\right)\left(x_{1}-x_{2}\right)\left(x_{1}-x_{3}\right)\left(x_{1}-x_{4}\right)} \\
L_{2}(x)=\frac{\left(x-x_{0}\right)\left(x-x_{1}\right)\left(x-x_{3}\right)\left(x-x_{4}\right)}{\left(x_{2}-x_{0}\right)\left(x_{2}-x_{1}\right)\left(x_{2}-x_{3}\right)\left(x_{2}-x_{4}\right)} \\
L_{3}(x)=\frac{\left(x-x_{0}\right)\left(x-x_{1}\right)\left(x-x_{2}\right)\left(x-x_{4}\right)}{\left(x_{3}-x_{0}\right)\left(x_{3}-x_{1}\right)\left(x_{3}-x_{2}\right)\left(x_{3}-x_{4}\right)} \\
L_{4}(x)=\frac{\left(x-x_{0}\right)\left(x-x_{1}\right)\left(x-x_{2}\right)\left(x-x_{3}\right)}{\left(x_{4}-x_{0}\right)\left(x_{4}-x_{1}\right)\left(x_{4}-x_{2}\right)\left(x_{4}-x_{3}\right)}
\end{array}\right.
$$

\section{THE MEASURED WORKPIECES}

There were performed the simulation investigations of coordinate measurements of selected products. The numerical research was done in order to verify the possibilities of using the developed algorithm of form deviation calculation. For each considered object there were extracted four free-form curves (Fig. 2).

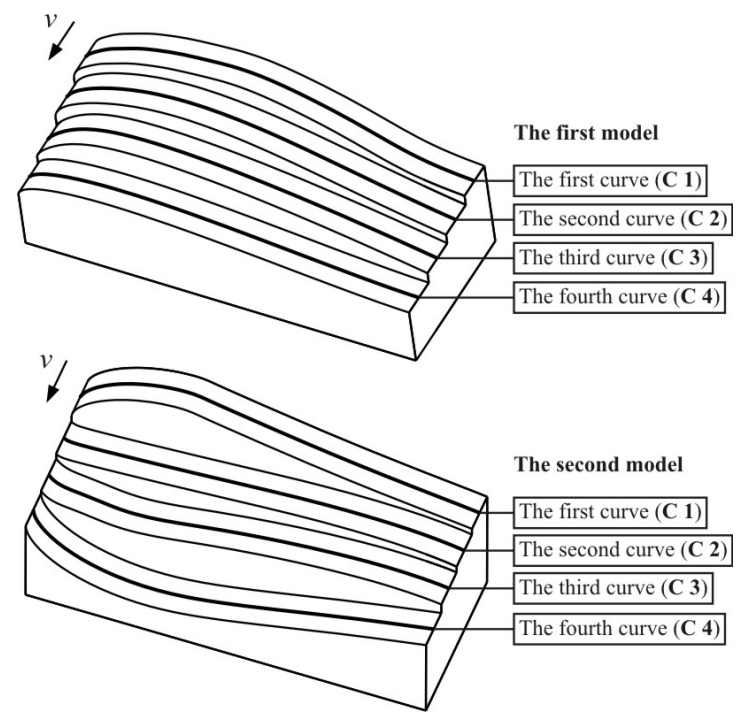

Fig. 2. The models of workpieces used during investigations

The measured workpieces had free-form surfaces with varying curvature. The nominal models were created with the use of the CATIA software and were imported into the measurement software in order to perform the simulation of coordinate measurements.

\section{THE SIMULATION INVESTIGATIONS}

The numerical investigations concerned coordinate measurements of considered objects (Fig. 2) on a coordinate measuring machine. The $2 \mathrm{D}$ curve measurement element was used during simulations. That element was chosen due to the lack of curvature along directions denoted as $v$ of analyzed curvilinear surfaces of measured products. There was used the uniform distribution of nominal and measured points along free-form surfaces of measured workpieces. The uniform distribution of nominal points along analyzed free-form profiles (denoted as C $\mathbf{1}-\mathbf{C ~ 4}$ ) was the result of using the $2 \mathrm{D}$ curve measurement element.

The used measurement element enables the equal distance between nominal points. The number of nominal points was equal 100 . The distances between measured points were equal: $(0.1,0.25,0.5,0.75,1)$ $\mathrm{mm}$. During conducted simulations the measured points were generated by using of the following dispersion: ( $\pm 0.01, \pm 0.05, \pm 0.1) \mathrm{mm}$ around nominal profiles. The dispersion of measured points results from the inaccuracy of manufacturing processes of measured workpieces and the inaccuracy of coordinate measurements of analyzed objects. The dispersion was applied in order to guarantee the randomness of results of measurements. In the case of the inaccuracy of machining processes randomly distributed points symbolized the real shape of the measured workpiece.

The investigations were conducted for three separate methods of form deviation calculation. The first two methods were described in the section 1 in detail. Those methods are based on nominal (the first method denoted as $\mathbf{N}$ in Tables 1 and 2) and measured (the second method - R) points at which local deviations are calculated. Additionally, in the case of the commercial method based on nominal points outliers were removed (NC). The way of removing outliers resulted from the algorithm of measurement data analysis which is available in the Calypso software. The outliers were removed in order to reduce the evaluated form deviations and make them correspond, as much as it is possible, to the assumed dispersion.

The third considered method was the new one, which is based on the interpolation process of groups of five neighboring points by using of the Lagrange (L) and Chebyshev $(\mathbf{C H})$ methods. In the case of the proposed algorithm of form deviation calculation outliers were removed as well. The selected results of numerical investigations for selected considered models of measured products, distances between measured points and their dispersion are presented in Tables 1 and 2. The results concern the form deviations of contour. The form deviations should be equal to twice the maximum local deviations. Therefore, the 
Table 1. The results of numerical investigations for the first model

\begin{tabular}{|c|c|c|c|c|c|c|c|}
\hline & $\begin{array}{c}\text { Distance } \\
\text { [mm] }\end{array}$ & C & $\begin{array}{c}\mathbf{N} \\
{[\mathrm{mm}]}\end{array}$ & $\begin{array}{c}\mathrm{NC} \\
{[\mathrm{mm}]}\end{array}$ & $\begin{array}{c}\mathbf{R} \\
{[\mathrm{mm}]}\end{array}$ & $\begin{array}{c}\text { CH } \\
{[\mathrm{mm}]}\end{array}$ & $\begin{array}{c}\mathbf{L} \\
{[\mathrm{mm}]}\end{array}$ \\
\hline \multirow{20}{*}{ 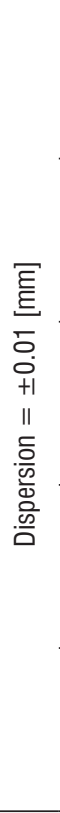 } & \multirow{4}{*}{0.1} & 1 & 0.0211 & 0.0211 & 0.0200 & 0.0249 & 0.0247 \\
\hline & & 2 & 0.0225 & 0.0225 & 0.0200 & 0.0235 & 0.0286 \\
\hline & & 3 & 0.0234 & 0.0234 & 0.0200 & 0.0247 & 0.0314 \\
\hline & & 4 & 0.0236 & 0.0236 & 0.0199 & 0.0249 & 0.0249 \\
\hline & \multirow{4}{*}{0.25} & 1 & 0.2947 & 0.0208 & 0.0200 & 0.0235 & 0.0235 \\
\hline & & 2 & 0.3559 & 0.0210 & 0.0200 & 0.0342 & 0.0278 \\
\hline & & 3 & 0.2409 & 0.0245 & 0.0200 & 0.0313 & 0.0312 \\
\hline & & 4 & 0.7923 & 0.0202 & 0.0200 & 0.0257 & 0.0257 \\
\hline & \multirow{4}{*}{0.5} & 1 & 1.0667 & 0.2126 & 0.0199 & 0.0329 & 0.0329 \\
\hline & & 2 & 1.1698 & 0.3232 & 0.0199 & 0.0192 & 0.0192 \\
\hline & & 3 & 0.7241 & 0.2015 & 0.0198 & 0.0247 & 0.0247 \\
\hline & & 4 & 2.0499 & 0.0223 & 0.0199 & 0.0284 & 0.0284 \\
\hline & \multirow{4}{*}{0.75} & 1 & 2.8797 & 0.0234 & 0.0199 & 0.0277 & 0.0277 \\
\hline & & 2 & 2.8222 & 0.0987 & 0.0193 & 0.0220 & 0.0220 \\
\hline & & 3 & 1.7194 & 0.0855 & 0.0199 & 0.0256 & 0.0257 \\
\hline & & 4 & 4.3864 & 0.8286 & 0.0200 & 0.0246 & 0.0246 \\
\hline & \multirow{4}{*}{1} & 1 & 5.2684 & 1.9676 & 0.0200 & 0.0264 & 0.0264 \\
\hline & & 2 & 4.9068 & 0.0901 & 0.0200 & 0.0279 & 0.0279 \\
\hline & & 3 & 2.8898 & 1.0925 & 0.0199 & 0.0270 & 0.0270 \\
\hline & & 4 & 8.3676 & 0.0958 & 0.0195 & 0.0266 & 0.0266 \\
\hline \multirow{20}{*}{ 它 } & \multirow{4}{*}{0.1} & 1 & 0.0985 & 0.0985 & 0.0999 & 0.1068 & 0.1076 \\
\hline & & 2 & 0.1183 & 0.1183 & 0.1000 & 0.1236 & 0.1259 \\
\hline & & 3 & 0.1027 & 0.1027 & 0.1000 & 0.1329 & 0.1326 \\
\hline & & 4 & 0.0961 & 0.0961 & 0.0999 & 0.1357 & 0.1357 \\
\hline & \multirow{4}{*}{0.25} & 1 & 0.3259 & 0.1123 & 0.0996 & 0.1207 & 0.1207 \\
\hline & & 2 & 0.2960 & 0.1010 & 0.0998 & 0.1579 & 0.1578 \\
\hline & & 3 & 0.1417 & 0.1417 & 0.0988 & 0.1324 & 0.1324 \\
\hline & & 4 & 0.7969 & 0.1167 & 0.0996 & 0.1586 & 0.1586 \\
\hline & \multirow{4}{*}{0.5} & 1 & 1.0544 & 0.2497 & 0.0999 & 0.1234 & 0.1234 \\
\hline & & 2 & 1.2080 & 0.2662 & 0.0999 & 0.1260 & 0.1260 \\
\hline & & 3 & 0.6985 & 0.1182 & 0.0997 & 0.1404 & 0.1404 \\
\hline & & 4 & 2.2498 & 0.1093 & 0.0992 & 0.1356 & 0.1356 \\
\hline & \multirow{4}{*}{0.75} & 1 & 2.8061 & 0.1157 & 0.0990 & 0.1170 & 0.1170 \\
\hline & & 2 & 2.8009 & 0.0983 & 0.0996 & 0.1332 & 0.1332 \\
\hline & & 3 & 1.6364 & 0.1164 & 0.0989 & 0.1223 & 0.1223 \\
\hline & & 4 & 4.5283 & 0.9895 & 0.0987 & 0.1494 & 0.1494 \\
\hline & & 1 & 5.1625 & 1.7798 & 0.0988 & 0.1076 & 0.1076 \\
\hline & & 2 & 4.9732 & 0.1259 & 0.1000 & 0.1168 & 0.1168 \\
\hline & & 3 & 2.8029 & 1.0193 & 0.0999 & 0.1424 & 0.1424 \\
\hline & & 4 & 8.6740 & 0.1130 & 0.0998 & 0.1503 & 0.1503 \\
\hline
\end{tabular}

form deviations should be equal respectively: $(0.02$, $0.1,0.2) \mathrm{mm}$.

\section{THE STATISTICAL ANALYSIS}

In the case of the new method of form deviation calculation, which can be used during coordinate measurements of workpieces composed of free-form
Table 2. The results of numerical investigations for the second model

\begin{tabular}{|c|c|c|c|c|c|c|c|}
\hline & $\begin{array}{c}\text { Distance } \\
{[\mathrm{mm}]}\end{array}$ & C & $\begin{array}{c}\mathbf{N} \\
{[\mathrm{mm}]}\end{array}$ & $\begin{array}{c}\text { NC } \\
{[\mathrm{mm}]}\end{array}$ & $\begin{array}{c}\mathbf{R} \\
{[\mathrm{mm}]}\end{array}$ & $\begin{array}{c}\text { CH } \\
{[\mathrm{mm}]}\end{array}$ & $\begin{array}{c}\mathbf{L} \\
{[\mathrm{mm}]}\end{array}$ \\
\hline \multirow{20}{*}{ 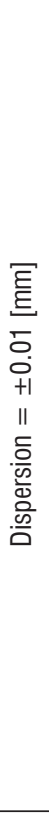 } & \multirow{4}{*}{0.1} & 1 & 0.0226 & 0.0226 & 0.0200 & 0.0271 & 0.0297 \\
\hline & & 2 & 0.0228 & 0.0228 & 0.0200 & 0.0311 & 0.0326 \\
\hline & & 3 & 0.0217 & 0.0217 & 0.0200 & 0.0319 & 0.0316 \\
\hline & & 4 & 0.0222 & 0.0222 & 0.0200 & 0.0279 & 0.0314 \\
\hline & \multirow{4}{*}{0.25} & 1 & 0.3532 & 0.0195 & 0.0198 & 0.0281 & 0.0281 \\
\hline & & 2 & 1.2584 & 0.0473 & 0.0200 & 0.0281 & 0.0282 \\
\hline & & 3 & 0.3015 & 0.0216 & 0.0200 & 0.0299 & 0.0298 \\
\hline & & 4 & 0.2324 & 0.0235 & 0.0200 & 0.0250 & 0.0250 \\
\hline & \multirow{4}{*}{0.5} & 1 & 0.9350 & 0.0216 & 0.0200 & 0.0227 & 0.0227 \\
\hline & & 2 & 2.7444 & 0.0211 & 0.0199 & 0.0264 & 0.0264 \\
\hline & & 3 & 1.0067 & 0.2815 & 0.0199 & 0.0276 & 0.0275 \\
\hline & & 4 & 0.7279 & 0.2128 & 0.0198 & 0.0292 & 0.0292 \\
\hline & \multirow{4}{*}{0.75} & 1 & 2.1434 & 0.1330 & 0.0200 & 0.0229 & 0.0229 \\
\hline & & 2 & 6.8376 & 0.0213 & 0.0199 & 0.0242 & 0.0246 \\
\hline & & 3 & 2.6960 & 0.2268 & 0.0200 & 0.0292 & 0.0292 \\
\hline & & 4 & 1.8546 & 0.0558 & 0.0199 & 0.0257 & 0.0257 \\
\hline & \multirow{4}{*}{1} & 1 & 3.7017 & 0.0690 & 0.0198 & 0.0305 & 0.0305 \\
\hline & & 2 & 6.9805 & 0.0241 & 0.0198 & 0.0252 & 0.0252 \\
\hline & & 3 & 5.0106 & 0.0208 & 0.0198 & 0.0246 & 0.0246 \\
\hline & & 4 & 3.3280 & 1.1266 & 0.0198 & 0.0281 & 0.0281 \\
\hline & \multirow{4}{*}{0.1} & 1 & 0.2110 & 0.2110 & 0.1999 & 0.2524 & 0.2524 \\
\hline & & 2 & 0.2382 & 0.2382 & 0.1998 & 0.2622 & 0.2623 \\
\hline & & 3 & 0.2028 & 0.2028 & 0.1999 & 0.2653 & 0.2449 \\
\hline & & 4 & 0.2132 & 0.2132 & 0.1998 & 0.2968 & 0.3040 \\
\hline & \multirow{4}{*}{0.25} & 1 & 0.5360 & 0.2122 & 0.1997 & 0.2650 & 0.2646 \\
\hline & & 2 & 1.1383 & 0.1986 & 0.1991 & 0.3100 & 0.3100 \\
\hline & & 3 & 0.2262 & 0.2262 & 0.1988 & 0.3212 & 0.3212 \\
\hline & & 4 & 0.3476 & 0.2348 & 0.1997 & 0.2322 & 0.2322 \\
\hline & \multirow{4}{*}{0.5} & 1 & 0.8834 & 0.2207 & 0.1994 & 0.2553 & 0.2553 \\
\hline & & 2 & 2.5056 & 0.2234 & 0.2000 & 0.2618 & 0.2618 \\
\hline & & 3 & 1.0715 & 0.1942 & 0.1973 & 0.2235 & 0.2235 \\
\hline & & 4 & 0.7557 & 0.2549 & 0.1986 & 0.2942 & 0.2942 \\
\hline & \multirow{4}{*}{0.75} & 1 & 1.9975 & 0.2786 & 0.1989 & 0.2868 & 0.2868 \\
\hline & & 2 & 7.7736 & 0.2377 & 0.1992 & 0.3145 & 0.3145 \\
\hline & & 3 & 2.8244 & 0.3449 & 0.1983 & 0.2491 & 0.2491 \\
\hline & & 4 & 1.9309 & 0.2232 & 0.1953 & 0.2268 & 0.2268 \\
\hline & \multirow{4}{*}{1} & 1 & 3.5546 & 0.2057 & 0.1959 & 0.2541 & 0.2541 \\
\hline & & 2 & 6.6456 & 0.3950 & 0.1991 & 0.2679 & 0.2679 \\
\hline & & 3 & 5.0134 & 0.2047 & 0.1999 & 0.2125 & 0.2125 \\
\hline & & 4 & 3.2780 & 0.2084 & 0.1999 & 0.2806 & 0.2806 \\
\hline
\end{tabular}

surfaces, the statistical analysis was conducted. The analysis was done in order to compare the values of computed deviations, which are estimated by using of two methods of interpolation - the Lagrange and the Chebyshev methods available in the proposed method of deviation estimation. Those interpolation methods are used in order to calculate the form deviations of free-form surfaces of measured products. 
The statistical analysis was conducted for the randomly chosen values of form deviations, which were selected from all results of numerical investigations obtained for the different measured workpieces, dispersion of corrected measured points and the distances between measured points. The measured objects, dispersion and distances used during the statistical analysis of gained results are shown in Tables 3 and 4. The analysis was done for deviations calculated at all nominal points of analyzed profiles.

Table 3. The results of statistical analysis for the first model

\begin{tabular}{lcccc}
\hline & Curve & $\begin{array}{c}\text { Dispersion } \\
{[\mathrm{mm}]}\end{array}$ & $\begin{array}{c}\text { Distance } \\
{[\mathrm{mm}]}\end{array}$ & $\begin{array}{c}\text { P-Value } \\
{[\%]}\end{array}$ \\
\hline \multirow{2}{*}{$\begin{array}{l}\text { The test of homogeneity } \\
\text { of mean }\end{array}$} & 1 & \pm 0.01 & 0.1 & 22.4 \\
\hline $\begin{array}{l}\text { The test of homogeneity } \\
\text { of variance }\end{array}$ & 1 & \pm 0.05 & 1 & 98.9 \\
\cline { 2 - 5 } & 4 & \pm 0.01 & 0.1 & 22.5 \\
\hline
\end{tabular}

Table 4. The results of statistical analysis for the second model

\begin{tabular}{lcccc}
\hline & Curve & $\begin{array}{c}\text { Dispersion } \\
{[\mathrm{mm}]}\end{array}$ & $\begin{array}{c}\text { Distance } \\
{[\mathrm{mm}]}\end{array}$ & $\begin{array}{c}\text { P-Value } \\
{[\%]}\end{array}$ \\
\hline \multirow{2}{*}{$\begin{array}{l}\text { The test of homogeneity } \\
\text { of mean }\end{array}$} & 3 & \pm 0.01 & 0.25 & 95.7 \\
\hline $\begin{array}{l}\text { The test of homogeneity } \\
\text { of variance }\end{array}$ & 3 & \pm 0.1 & 0.1 & 97.9 \\
\cline { 2 - 5 } & 3 & \pm 0.01 & 0.25 & 97.0 \\
\hline
\end{tabular}

In the first stage of analysis the comparison tests of arithmetical means of deviations calculated with the use of the Lagrange and Chebyshev methods were conducted. The null hypothesis in that stage of analysis concerned the equality of arithmetical means of deviations estimated using two above mentioned interpolation methods. The analysis of variance (known as ANOVA) was used. The level of significance was set at $5 \%$. The critical values $p$ obtained on the basis of statistical analysis are presented in Tables 3 and 4 . All critical values are greater than the assumed level of significance. It means that there is no basis to reject the null hypothesis of equality of arithmetical means of deviations calculated by using of two considered algorithms of interpolation. Therefore, the results gained by using of considered interpolation methods do not differ significantly from each other.

In the next stage of statistical analysis the tests of homogeneity of variance were conducted. The Levene's test was used. The used test can be implemented when there is no certainty about the normal distribution of measured deviations at all nominal points. The null hypothesis concerned the equality of variances, which were calculated when using two interpolation algorithms based on the Lagrange and Chebyshev methods. The level of significance was equal $5 \%$, similarly to the first stage of analysis. The statistical tests gave the critical values greater than the used level of significance as well. The results of calculations are presented in Tables 3 and 4. Therefore, there is no basis to reject the null hypothesis of equality of variances, which were calculated by using of considered interpolation methods available in the proposed method of form deviation calculation.

\section{THE EXPERIMENTAL INVESTIGATIONS}

The experimental investigations concerned real contact coordinate measurements of the profile $\mathbf{C} \mathbf{1}$ in the case of the first product (Fig. 2) by using of the coordinate measuring machine ACCURA II equipped with the measuring probe VAST XT (Fig. 3) and the Calypso inspection software. The selected accuracy parameters of the applied measuring system are the following:

$$
\begin{aligned}
& \text { - } E_{L, M P E}=1.6+\mathrm{L} / 333 \mu \mathrm{m}, \\
& \text { - } P_{F T U, M P E}=1.7 \mu \mathrm{m}, \\
& \text { - } \quad M P E_{T i j}=2.5 \mu \mathrm{m}, \\
& \text { - } M P T_{\tau i j}=50 \mathrm{~s} .
\end{aligned}
$$

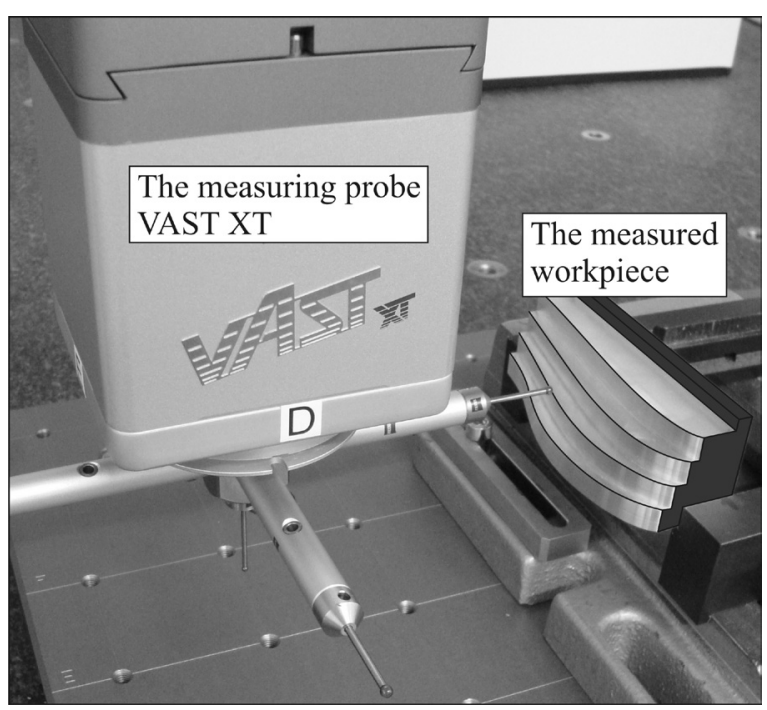

Fig. 3. The coordinate measurements of the first object

The measured product was made of aluminum alloy and machined by using of the DMU 100 monoBLOCK CNC machine tool. Similar to the simulation studies there was used the uniform distribution of nominal and measured points. The measurements were performed with the use of different 
scanning velocities, denoted as $v_{s}$ and distances between measured points, denoted as $d$. There were used the following measurement parameters:

- $v_{s}=10 \mathrm{~mm} / \mathrm{s}, 20 \mathrm{~mm} / \mathrm{s}, 30 \mathrm{~mm} / \mathrm{s}$,

- $\quad d=(0.5,1,1.5,2,2.5,3) \mathrm{mm}$.

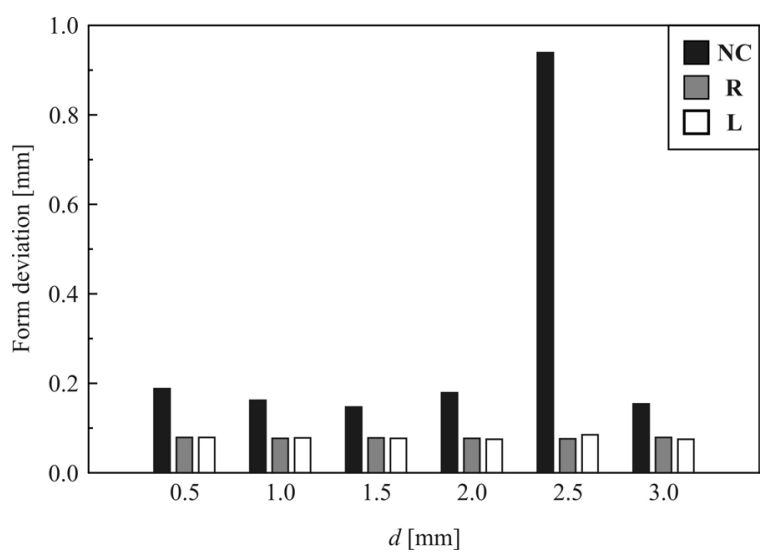

Fig. 4. The results of experimental research for $v_{s}=10 \mathrm{~mm} / \mathrm{s}$

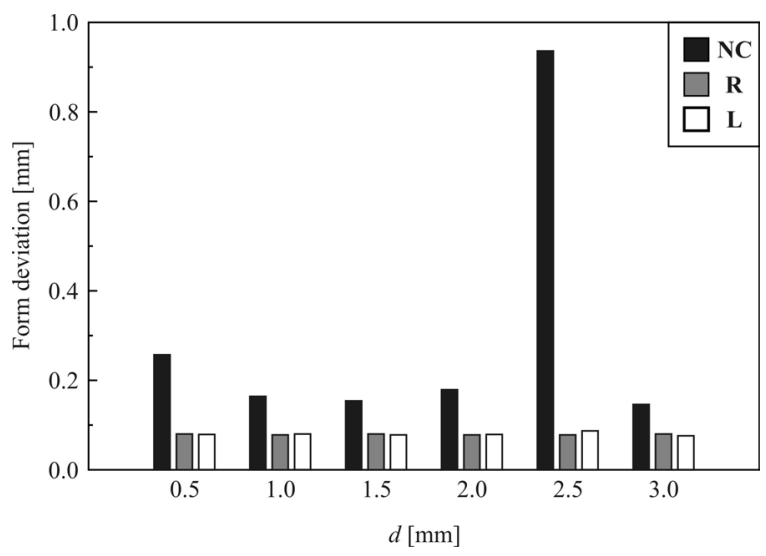

Fig. 5. The results of experimental research for $v_{S}=20 \mathrm{~mm} / \mathrm{s}$

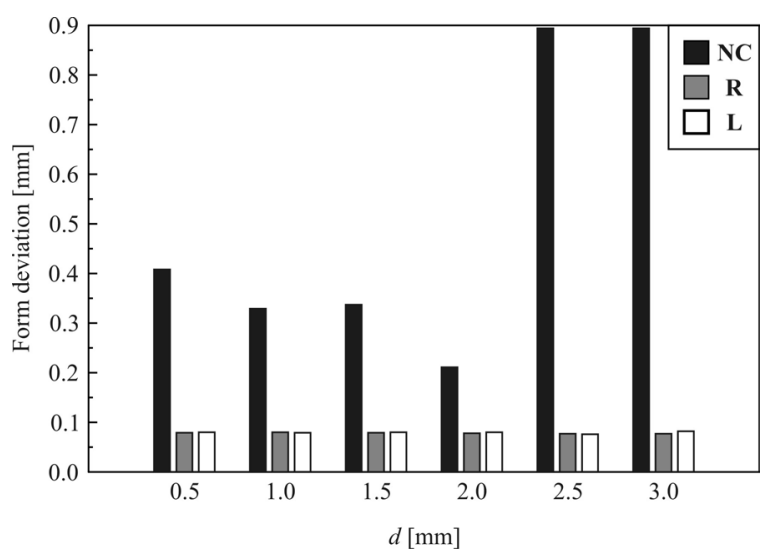

Fig. 6. The results of experimental research for $v_{s}=30 \mathrm{~mm} / \mathrm{s}$

Moreover, the coordinate measurements were conducted by using of the same algorithms of form deviation calculation like those used during the simulation investigations. They were based on nominal points (NC), measured points (R) and the Lagrange interpolation method $(\mathbf{L})$. The method $\mathbf{L}$ was applied during the experimental research because, based on the results of statistical analysis, there are no significant differences between two analyzed interpolation algorithms. The results of performed experimental research are presented in Figs. 4 to 6 .

\section{DISCUSSION}

The new method of form deviation calculation was verified during the simulation and experimental investigations. It should be expected that the calculated form deviations of analyzed free-form surfaces of products in numerical investigations should correspond to the assumed dispersion of measured points. However, the experiments showed that the values of a form deviation similar to the values of a range were gained only in the case of the second method of form deviation calculation, which is based on measured points. In the case of other considered methods, which use nominal points to calculate form deviations of curvilinear surfaces, values of a form deviation exceed values of a range of generated measured points.

The results of conducted numerical research indicate that there is no influence of the distance between measured points on the values of a form deviation in the case of the proposed method of form deviation calculation. That is the advantage of the new algorithm. When using the first method of form deviation calculation, available in the commercial software of e.g. coordinate measuring machines, the calculated form deviations increase with decreasing the number of measured points. In the case of the first method removing outliers causes lower values of a form deviation but not correspond to the dispersion in all analyzed cases.

The conducted statistical analysis of selected results of simulation investigations shows that there are no significant differences between two interpolation methods available in the proposed method of form deviation calculation when comparing the gained values of a deviation.

The experimental investigations confirm the results of numerical research. The form deviations calculated by using of the proposed method are lower than the values of a deviation calculated with the use of the method NC available in the commercial inspection software. The deviations calculated using the algorithm based on the Lagrange interpolation 
method are close to the values of a deviation gained by using of the method $\mathbf{R}$. Based on the results of numerical research, the method $\mathbf{R}$ can be considered as the reference one. Moreover, the deviations calculated by using of the method NC are dependent largely on the applied scanning velocities and distances between measured points.

\section{CONCLUSIONS}

In the article the new method of form deviation calculation was presented. The proposed algorithm is based on the interpolation process of corrected measured points with the use of the Lagrange and Chebyshev methods. The proposed method of form deviation calculation achieved better results of investigations than the method based on nominal points in the Calypso software.

The advantage of the proposed method is the possibility of choosing the appropriate algorithm of interpolation of measured points. In the case of the considered measurement software a user cannot select other methods of interpolation of corrected measured points besides the default available algorithm.

The additional advantage of the developed algorithm is that it can be implemented in most measurement software. In the case of the Calypso software the implementation can be realized by using of the PCM module. The user is able to apply the external user-defined procedure and execute user programs under its control. Such approach gives the user enhanced possibilities to analyze the results of coordinate measurements.

\section{REFERENCES}

[1] Mansour, G. (2014). A developed algorithm for simulation of blades to reduce the measurement points and time on coordinate measuring machine (CMM). Measurement, vol. 54, p. 51-57, Dol:10.1016/J.measurement.2014.03.046.

[2] Poniatowska, M. (2012). Deviation model based method of planning accuracy inspection of free-form surfaces using CMMs. Measurement, vol. 45, no. 5, p. 927-937, D0l:10.1016/j.measurement.2012.01.051.

[3] Uhlmann, E., Abackerli, A.J., Schützer, K., Lepikson, H.A., Helleno, A.L., Papa, M.C.O., del Conte, E.G., Mewis, J. (2014).
Simulation and analysis of error impact on freeform surface milling. The International Journal of Advanced Manufacturing Technology, vol. 70, no. 1, p. 607-620, Dol:10.1007/s00170013-5280-7.

[4] Cheung, C.F., Kong, L.B., Ren, M.J., Whitehouse, D., To, S. (2012). Generalized form characterization of ultra-precision freeform surfaces. CIRP Annals - Manufacturing Technology, vol. 61, no. 1, p. 527-530, Dol:10.1016/j.cirp.2012.03.015.

[5] Savio, E., De Chiffre, L., Schmitt, R. (2007). Metrology of freeform shaped parts. CIRP Annals - Manufacturing Technology, vol. 56, no. 2, p. 810-835, Dol:10.1016/j. cirp.2007.10.008.

[6] Fang, F. Z., Zhang, X. D., Weckenmann, A., Zhang, G. X., Evans, C. (2013). Manufacturing and measurement of freeform optics. CIRP Annals - Manufacturing Technology, vol. 62, no. 2, p. 823-846, D0l:10.1016/j.cirp.2013.05.003.

[7] Savio, E., Hansen, H.N., De Chiffre, L. (2002). Approaches to the calibration of freeform artefacts on coordinate measuring machines. CIRP Annals - Manufacturing Technology, vol. 51, no. 1, p. 433-436, D0l:10.1016/S0007-8506(07)61554-6.

[8] Mehrad, V., Xue, D., Gu, P. (2013). Prediction of surface reconstruction uncertainties for freeform surface inspection. Measurement, vol. 46, no. 8, p. 2682-2694, D0l:10.1016/j. measurement.2013.04.025.

[9] EN ISO 15530:2011. Geometrical product specifications (GPS) - Coordinate measuring machines (CMM): Technique for determining the uncertainty of measurement - Part 3: Use of calibrated workpieces or measurement standards. International Organization for Standardization, Geneva.

[10] Liang, S.R., Lin, A.C. (2002). Probe-radius compensation for 3D data points in reverse engineering. Computers in Industry, vol. 48, no. 3, p. 241-251, D0l:10.1016/S0166-3615(02)00038-6.

[11] Vosniakos, G.C., Giannakakis, T. (2003). Reverse engineering of simple surfaces of unknown shape with touch probes: scanning and compensation issues. Proceedings of the Institution of Mechanical Engineers, Part B: Journal of Engineering Manufacture, vol. 217, no. 4, p. 563-568, DOI:10.1243/095440503321628233.

[12] Wozniak, A., Balazinski, M., Mayer, R. (2007). Application of fuzzy knowledge base for corrected measured point determination in coordinate metrology. Annual Meeting of the North American Fuzzy Information Processing Society, p. 135139, D0I:10.1109/NAFIPS.2007.383825.

[13] Woźniak, A., Mayer J.R.R., Bałaziński, M. (2009). Stylus tip envelop method: corrected measured point determination in high definition coordinate metrology. The International Journal of Advanced Manufacturing Technology, vol. 42, no. 5, p. 505514, D0I:10.1007/s00170-008-1615-1.

[14] Calypso. Carl Zeiss, software documentation. (2010), Carl Zeiss Industrielle Messtechnik, Oberkochen. 